\title{
Categorical Speech Processing in Broca's Area: An fMRI Study Using Multivariate Pattern-Based Analysis
}

\author{
Yune-Sang Lee, ${ }^{1}$ Peter Turkeltaub, ${ }^{2}$ Richard Granger, ${ }^{1}$ and Rajeev D. S. Raizada ${ }^{3}$ \\ ${ }^{1}$ Department of Psychological and Brain Sciences, Dartmouth College, Hanover, New Hampshire 03755, ${ }^{2}$ Neurology Department, Georgetown University, \\ Washington, DC, 20057 and ${ }^{3}$ Neukom Institute, Dartmouth College, Hanover, New Hampshire 03755
}

\begin{abstract}
Although much effort has been directed toward understanding the neural basis of speech processing, the neural processes involved in the categorical perception of speech have been relatively less studied, and many questions remain open. In this functional magnetic resonance imaging (fMRI) study, we probed the cortical regions mediating categorical speech perception using an advanced brain-mapping technique, whole-brain multivariate pattern-based analysis (MVPA). Normal healthy human subjects (native English speakers) were scanned while they listened to 10 consonant-vowel syllables along the /ba/-/da/ continuum. Outside of the scanner, individuals' own category boundaries were measured to divide the fMRI data into /ba/ and /da/ conditions per subject. The whole-brain MVPA revealed that Broca's area and the left pre-supplementary motor area evoked distinct neural activity patterns between the two perceptual catego$\operatorname{ries}(/ \mathrm{ba} / \mathrm{vs} / \mathrm{da} /$ ). Broca's area was also found when the same analysis was applied to another dataset (Raizada and Poldrack, 2007), which previously yielded the supramarginal gyrus using a univariate adaptation-fMRI paradigm. The consistent MVPA findings from two independent datasets strongly indicate that Broca's area participates in categorical speech perception, with a possible role of translating speech signals into articulatory codes. The difference in results between univariate and multivariate pattern-based analyses of the same data suggest that processes in different cortical areas along the dorsal speech perception stream are distributed on different spatial scales.
\end{abstract}

\section{Introduction}

One of the core aspects of speech perception is mapping complex time-varying acoustic signals into discrete speech units. The "categorical speech perception" phenomenon was experimentally demonstrated in the 1950s, whereby synthetic syllables along the continuum between prototypes (e.g., /ba/vs /da/) were perceived categorically despite their linear acoustic variations (Liberman et al., 1957). According to one of the most influential speech theories (Liberman and Mattingly, 1985), this perceptual categorization of incoming auditory speech occurs because articulatory gestures serve as the brain's representations of speech sounds, and speech is perceived by mapping continuous auditory signals onto discrete articulatory gestures. More recent theories based on converging evidence from lesion, neuroimaging, and brain stimulation studies (for review, see Devlin and Aydelott, 2009) discount the role of articulatory gestures as perceptual representations, postulating instead that speech percepts and motor codes interact via feedforward and feedback connections (Hickok and Poeppel, 2007; Rauschecker and Scott, 2009).

\footnotetext{
Received July 22, 2011; revised Jan. 24, 2012; accepted Jan. 27, 2012.

Author contributions: Y.S.L., R.G., and R.D.S.R. designed research; Y.S.L. and R.D.S.R. performed research; Y.S.L. and R.D.S.R. analyzed data; Y.S.L., P.T., R.G., and R.D.S.R. wrote the paper.

This work was supported by grants from the Office of Naval Research. We thank Yale Cohen, Tilbe Goksun, David Kraemer, and Kate Onsgard for their helpful comments on the earlier version of the manuscript. Special thanks go to the Penn Postdoc Editors Association for proofreading.

The authors declare no financial conflicts of interest.

Correspondence should be addressed to Dr. Yune-Sang Lee, Center for Cognitive Neuroscience, 3710 Goddard Labora-

tory, Hamilton Walk, University of Pennsylvania, Philadelphia, PA 19104. E-mail:yslee@mail.med.upenn.edu.

R. D. S. Raizada's present address: Department of Human Development, Cornell University, Ithaca, NY.

DOI:10.1523/JNEUROSCI.3814-11.2012

Copyright $\odot 2012$ the authors $\quad 0270-6474 / 12 / 323942-07 \$ 15.00 / 0$
}

This auditory-motor integration is thought to be achieved along a dorsal stream speech network, running from primary auditory cortex via posterior superior temporal gyrus (STG) and the inferior parietal lobule to the posterior frontal lobe (Hickok and Poeppel, 2007). The left posterior STG has been implicated in categorical phoneme perception by functional imaging (Husain et al., 2006; Desai et al., 2008; Hutchison et al., 2008) and intracranial human electrophysiology studies (Chang et al., 2010). The supramarginal gyrus (SMG) has also been implicated in categorical speech perception. Raizada and Poldrack (2007) found that the SMG evoked amplified neural activity in response to between-category phoneme pairs along the syllabic continuum of /ba/ and /da/. Furthermore, a recent meta-analysis revealed that the SMG and angular gyrus were consistently activated in fMRI studies of categorical speech perception (Turkeltaub and Coslett, 2010). Thus, posterior nodes of the dorsal speech pathway involved in spectrotemporal analysis of auditory signals, phonological processing, and sensorimotor interface have been clearly implicated in categorical perception of speech. In contrast, findings in frontal articulatory coding areas (posterior inferior frontal gyrus, premotor cortex, anterior insula) have occasionally been reported in studies of categorical speech perception (Myers et al., 2009), but are far less consistent.

We hypothesized that frontal articulation areas are involved in categorical speech perception, but that they may be invisible to subtraction-based fMRI analysis if complex articulatory gestures are represented not by different levels of activity within single voxels, but by differential neural activity patterns within a region of cortex. As such, the present fMRI study used a relatively new mapping strategy, the searchlight analysis (Kriegeskorte et al., 


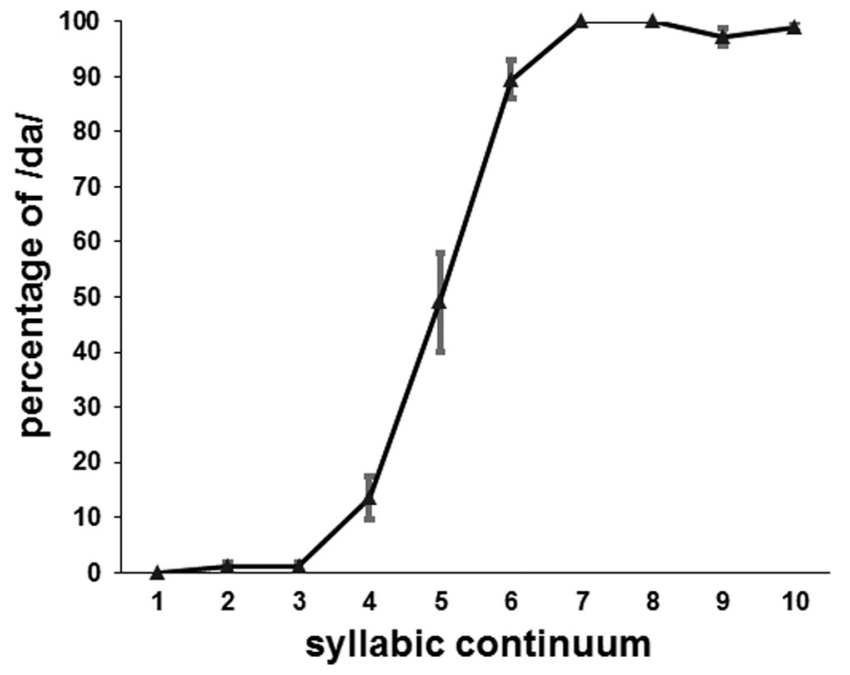

Figure 1. The average identification curve from the 13 participants. The $x$-axis shows the 10 -step syllabic continuum in numbers between 1 (/ba/ end point) and 10 (/da/ end point). The acoustic distance between successive steps is identical. The $y$-axis shows the /da/ response frequency percentage at each point; error bars indicate SEs.

2006), to explore the neuroanatomical basis of categorical speech perception. This technique, based on multivariate patternanalysis (MVPA) (Haxby et al., 2001; Pereira et al., 2009), probes local information of neural pattern differences across different conditions by moving a searchlight unit on a voxel-by-voxel basis. Some fMRI studies have used MVPA to examine speech perception (Formisano et al., 2008; Okada et al., 2010; Raizada et al., 2010; Kilian-Hütten et al., 2011). Most, however, examined the neural activity patterns exclusively within superior temporal regions. The searchlight procedure allowed us to examine the entire brain.

\section{Materials and Methods \\ Subjects}

Fifteen right-handed native English speakers without reported hearing difficulties or neurological disorders (10 males/5 females; ages, 19-34 years) were recruited from the Dartmouth College community. Two were excluded (one fell asleep during scanning, and one did not have a clear categorical boundary in the behavioral experiment), leaving 13 subjects total. Consent was obtained from all subjects as approved by the Committee for the Protection of Human Subjects at Dartmouth College.

\section{Stimuli}

Ten synthesized phonemes (duration of each: $300 \mathrm{~ms}$ ) along the /ba/-/da/ continuum were created by varying the second and third formant using a SenSyn Klatt Synthesizer (Sensimetrics), as described by Raizada and Poldrack (2007). Each phoneme along the continuum was referred to by number, with 1 being the stimulus of clear /ba/ and 10 being /da/ (Fig. 1).

\section{Experimental procedures}

fMRI experiment. During scanning, subjects listened to the stimuli while performing a nonphonetic task designed to maintain alertness, in which they were required to indicate a quieter stimulus that was presented in the catch trial block by pressing a button with their right hand.

Behavioral experiment. After the fMRI experiment, subjects were behaviorally tested to measure a categorical boundary on the 10 syllabic tokens that were previously presented in the scanner. Each syllabic sound was presented eight times, and subjects were required to indicate whether they perceived /ba/ or /da/ by button-press. The boundary was defined as the $50 \%$ crossover point between perception of $/ \mathrm{ba} / \mathrm{and} / \mathrm{da} /$ on each subject's psychometric curve and was used to define binary classes (/ba/ $\mathrm{vs} / \mathrm{da} /$ ) to label neural data per subject.

\section{fMRI scanning}

A Philips Intera Achieva 3T scanner was used with a standard EPI BOLD pulse sequence and a clustered volume acquisition. The parameters were as follows: repetition time (TR), $3 \mathrm{~s}$; acquisition time, $1.88 \mathrm{~s}$; silent gap, $1.12 \mathrm{~s} ; 560 \mathrm{~ms}$ interval between stimuli and scanner-noise onset/offset; 32 slices; $3 \times 3 \mathrm{~mm}$ within plane resolution, $4 \mathrm{~mm}$ thick slices with a $0.5 \mathrm{~mm}$ skip; interleaved slice-ordering. Each stimulus was presented in the middle of the silent gap before the next onset of EPI acquisition. In the scanner, sounds were played via high-fidelity MR compatible headphones (MR Confon). The fMRI scans were subdivided into five runs with 185 volume acquisitions per run. A block design was used, with one of the 10 phonemes repeatedly presented five times in each block. Between the blocks were rest periods lasting $15 \mathrm{~s}$ ( 5 TRs). The order of the blocks was pseudorandom and counterbalanced across subjects. There were 18 stimulus blocks per run, making 90 blocks total across the five runs. Of these, 10 contained quieter catch-trials that were excluded from subsequent analysis. The remaining 80 blocks consisted of eight blocks for each of the 10 stimuli along the /ba/-/da/ continuum.

\section{fMRI data (searchlight procedure) analysis methods}

fMRI data were preprocessed using SPM5 and MATLAB 2009b. All images were realigned to the first EPI and spatially normalized into Montreal Neurological Institute (MNI) standard stereotactic space (ICBM152 EPI template) with preserved original voxel size. After preprocessing, fMRI time courses of all voxels were extracted from unsmoothed images. Subsequently, these raw signals were high-pass filtered with a $300 \mathrm{~s}$ cutoff to remove slow drifts caused by the scanner, and standardized across entire runs using the $z$-score to normalize intensity differences among runs. When the data were subsequently entered into a classifier in pattern-based analysis, the TRs corresponding to each syllabic stimulus were labeled as /ba/ or / da/ by using the stimulus blocks' presentation times. For each stimulus-type, the time courses of its stimulus-presentation blocks were convolved with a canonical hemodynamic response function (HRF), thereby producing a regressor for that class of stimuli. The mean value across all time points of the regressor was then calculated. A time point was assigned as belonging to the given stimulus class if the value of the regressor at that time point was greater than this overall mean value.

For the primary searchlight analysis, local searchlight spheres consisting of a center voxel and its neighborhood within a three-voxel radius were constructed throughout the entire brain $(\sim 30,000 \mathrm{U})$. Then, in each searchlight sphere, a binary classification was performed using the Gaussian Naive Bayes classifier (Pereira et al., 2009). The classifier was initially trained by a subset of datasets (training set) to build a model that appropriately set the boundary between the neural activities that were associated with a perceptual category of /ba/ and /da/. Then, this model was applied to the remaining datasets (testing set) for validation, wherein the accuracy of classification test was gained by calculating how many times the classifier correctly predicted time points corresponding either to $/ \mathrm{ba} /$ or to $/ \mathrm{da} /$ in the unseen dataset (chance level, $50 \%$ ). To avoid a bias caused by a particular training/testing set, this procedure was repeated five times, such that signals from four scanning runs were served as a training set and one remaining run served as a testing set (i.e., fivefold cross-validation). The classification accuracy for each searchlight sphere was averaged across the testing set results of the five training/testing combinations and stored in each voxel of an output image for each subject (for more details, see Kriegeskorte et al., 2006).

After output images were acquired by searchlight analysis, baseline corrections in voxel values were made via two steps. First, the chancelevel accuracy, i.e., 0.5, was subtracted from an accuracy stored in each voxel of the output images. Next, the mean of these accuracy values in the output image was subtracted from each voxel's value, such that the resulting output image for each subject had a mean of zero. This baselinecorrection procedure has two advantages. First, it guards against false discovery (i.e., more conservative than the standard procedure with no baseline correction). Second, it helps to normalize different accuracy ranges across subjects (i.e., reducing the variance in the signal at a group level, which in turn increases the $t$-statistical power at the group level). The adjusted accuracy map was submitted to the random-effects analysis 
and threshold of $p$ (cluster-wise corrected) $<0.05$ in combination with $p$ (voxelwise uncorrected) $<0.001$ was applied throughout the searchlight analyses. For the random effects analyses on both current and old datasets, SPM8 was used instead to take advantage of its newly added feature, cluster-level correction based on false discovery rate in the updated version of SPM.

A new analysis, using a searchlight approach, of the Raizada and Poldrack 2007 dataset

Procedures were identical to those described above (fMRI data (searchlight procedure) analysis methods), except that the smoothed data of 12 subjects were used because unsmoothed data were not available (for details, see Raizada and Poldrack, 2007). In this 2007 study, stimuli were presented in pairs of two types using event-related design: identical pairs, where one token on the 10-step /ba/-/da/ continuum was presented twice in succession (e.g., 4-then-4); and three-step pairs, in which the two stimuli were three steps apart along the continuum (e.g., 4-then-7). In the present analysis, only the identical pairs were analyzed,

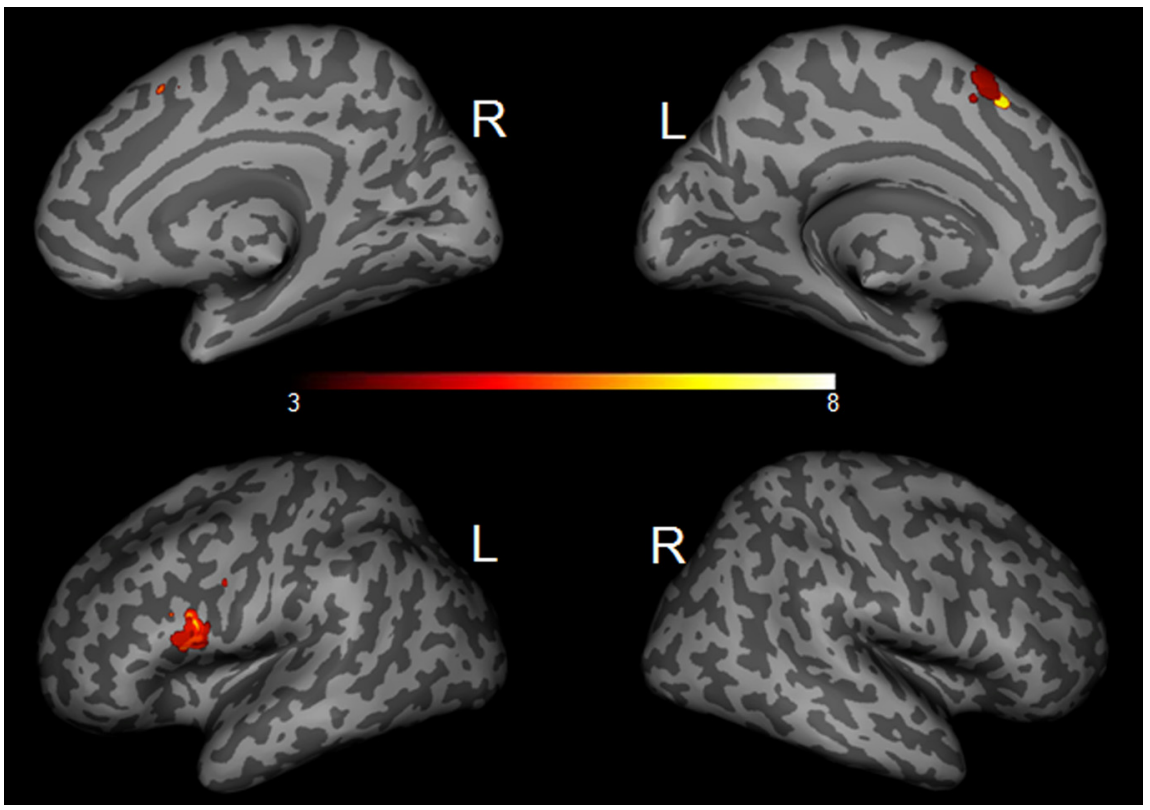

Figure 2. Broca's area (MNI $x, y, z=-54,12,22)$ and pre-SMA (MNI $x, y, z=-3,18,50)$ clusters were overlaid on the surface-inflated rendering brain using SPM surfrend toolbox (http://spmsurfrend.sourceforge.net/). R, right; $L$, left. as these are directly comparable to the uniform blocks of stimuli that were used in the newly acquired data.

\section{Conventional univariate GLM analysis}

For comparison purposes, we also performed standard univariate GLM analyses of the data, investigating the following four contrasts: /ba/ $/ \mathrm{da} /, / \mathrm{da} /-/ \mathrm{ba} /$, and, as simple controls, all-speech - baseline and baseline - all-speech. These GLM analyses were applied to the smoothed images of the current dataset for each subject using SPM5. At the firstlevel analysis, the fMRI time courses corresponding to each block of stimulus were convolved with canonical HRF using the box-car design. Additionally, the six motion parameters, as well as the onset of button responses, were modeled; they were later regressed out as effects of noninterest. Contrast images from each subject's first-level analysis were submitted to the second-level random effects for group analysis.

\section{Results}

\section{Behavioral results}

As expected, nearly all subjects demonstrated a sharp category boundary near the middle of the syllabic continuum between /ba/ and /da/ (Fig. 1). However, one subject did not perceive the syllabic continua in a categorical manner (i.e., linear trend) and was later discarded from further analysis. This subject was a native English speaker, but spent several years during childhood in Hong Kong, which may have affected the behavioral results. The category boundary varied slightly among the subjects (Fig. 1, error bars).

\section{fMRI}

MVPA

The whole-brain searchlight analysis equipped with three-voxel radius spheres revealed two left frontal regions, namely, the preSMA and the pars opercularis within Broca's area (Figs. 2, 3a; Table 1). No cortical regions within the temporal or parietal lobes were significant. To further validate these results, we reanalyzed previously published adaptation fMRI data (Raizada and Poldrack, 2007) using the same MVPA procedures. Despite several differences between these two datasets (design, number of available trials, degree of smoothness, participating subjects), the left pars opercularis again was significant at a matched threshold (Fig. $3 c$,
Table 1); no other significant areas were identified. Note that the previous dataset implicated the left SMG in categorical phoneme perception using the adaptation fMRI paradigm (Fig. $3 d$, Table 1). However, this area did not emerge when the MVPA procedure was used on either dataset $\left(t_{12}=1.3, t_{11}=0, p=\right.$ n.s; for new and old datasets, respectively).

To confirm that the activity identified in the MVPA analyses related to individual subjects' percepts rather than acoustic differences between items at each end of the continuum, we performed another searchlight analysis with categories binarized based on the absolute midpoint along the continuum, disregarding each subject's category boundary. As a result, no significant areas were found even at a relaxed threshold [ $p$ (voxelwise uncorrected $)<0.01$.

The difference between the searchlight analysis results and the previous univariate results could be interpreted as a difference in the spatial scale of processing in different areas of the network (intravoxel in the SMG vs intervoxel in the pars opercularis). This theory raised the possibility that processing in other areas of the speech perception network might be distributed over regions of cortex too large to be detected using a three-voxel searchlight. To explore this possibility, we performed additional searchlights analyses with a systematic manipulation of the searchlight size parameter. At a radius of eight-voxels, a significant cluster emerged within the left STG in the new dataset at a matched threshold (Fig. 3d, Table 1). Interestingly, the pars opercularis and pre-SMA were no longer significant in that larger searchlight analysis. Nevertheless, the old dataset did not yield significant temporal clusters in any of the additional analyses.

\section{Conventional univariate analysis}

The comparison of all sounds versus resting blocks yielded significant clusters within the expected cortical regions along the speech network, including bilateral temporal lobes, left parietal region, and frontal regions (data not shown). In contrast, a complementary univariate comparison of items perceived by each subject as /ba/ versus /da/ revealed no significant areas even at a relaxed threshold $[p$ (voxelwise uncorrected) $<0.01)$ ], confirm- 

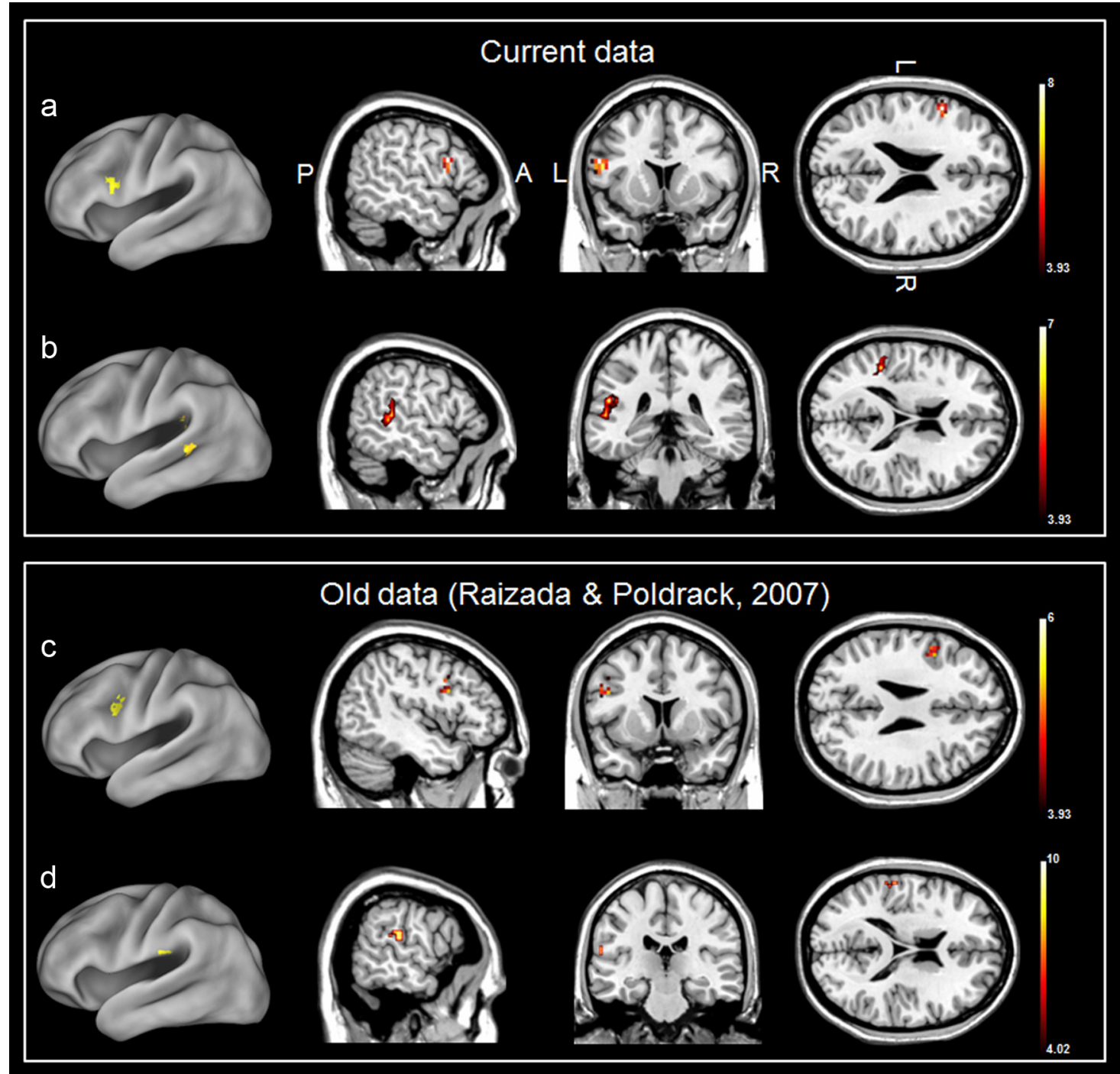

Figure 3. Top, Results from new dataset. $\boldsymbol{a}, \boldsymbol{b}$, Broca's area $(\boldsymbol{a}, \mathrm{MNI} x, y, z=-54,12,22)$ and left STG ( $\boldsymbol{b})$ identified by the searchlight analyses equipped with three- and eight-voxel radii of local spheres, respectively. $\boldsymbol{c}, \boldsymbol{d}$, Results from Raizada and Poldrack (2007). $\boldsymbol{c}$, Broca's area (MNI $x, y, z=-45,9,27$ ) identified by the searchlight analysis with a three-voxel radius of local sphere. $\boldsymbol{d}$, The left SMG (MNI $x, y, z=-60,-24,18$ ) previously identified by adaptation analysis (Raizada and Poldrack, 2007). The color bars indicate $t$-vales of the group random-effects analysis. P, posterior; $A$, anterior; $L$, left; $R$, right.

Table 1. Cortical loci involved in categorical speech perception

\begin{tabular}{|c|c|c|c|c|c|c|c|c|c|}
\hline \multirow[b]{2}{*}{ Region name } & \multirow[b]{2}{*}{ HEM } & \multirow[b]{2}{*}{ BA } & \multicolumn{3}{|c|}{ MNI coordinates } & \multirow[b]{2}{*}{$z$ value } & \multirow[b]{2}{*}{$t$ value } & \multirow[b]{2}{*}{ Cluster } & \multirow[b]{2}{*}{ Dataset } \\
\hline & & & $x$ & $y$ & $z$ & & & & \\
\hline \multicolumn{10}{|l|}{ MVPA (3-voxel radius searchlight) } \\
\hline PreSMA & L & 6 & -3 & 18 & 50 & 4.32 & 6.94 & 12 & Current fMRI data \\
\hline Pars opercularis & L & 44 & -54 & 12 & 22 & 4.09 & 6.25 & 24 & Current fMRI data \\
\hline Pars opercularis & $\mathrm{L}$ & 44 & -45 & 9 & 27 & 3.68 & 5.35 & 25 & Raizada and Poldrack (2007) \\
\hline MVPA (8-voxel radius searchlight) & & & & & & & & & \\
\hline $\begin{array}{l}\text { Superior temporal gyrus/sulcus } \\
\text { Adaptation }\end{array}$ & L & 22 & -51 & -36 & 23 & 4.21 & 6.60 & 42 & Current fMRI data \\
\hline $\begin{array}{l}\text { Adaptation } \\
\text { Supramarginal gyrus }\end{array}$ & $\mathrm{L}$ & 40 & -60 & -24 & 18 & 4.78 & 9.16 & 30 & Raizada and Poldrack (2007) \\
\hline
\end{tabular}

The statistical maps were significant at voxel-wise uncorrected $p<0.001$ and clusterwise corrected $p<0.05$.

HEM, Hemisphere; BA, approximate Brodmann area; L, left; $R$, right.

ing that MVPA clusters did not represent a simple difference in voxelwise activation intensity.

\section{Discussion}

Although the neural basis of speech perception has been extensively studied in the auditory neuroimaging field, relatively little evidence has been garnered as to how speech sounds are sorted into discrete categories (Turkeltaub and Coslett, 2010). Previous evidence, based largely on standard univariate fMRI analysis, has mainly suggested roles for posterior temporal and inferior parietal regions in categorical speech perception (Celsis et al., 1999; Zevin and McCandliss, 2005; Husain et al., 2006; Raizada and 
Poldrack, 2007; Desai et al., 2008; Hutchison et al., 2008; Chang et al., 2010; Zevin et al., 2010; Kilian-Hütten et al., 2011). Nevertheless, theories implicating sensorimotor mapping in speech sound categorization (Devlin and Aydelott, 2009) suggest frontal regions should be involved as well. In line with this hypothesis, the present MVPA study revealed that two frontal regions, namely, Broca's area and left pre-SMA, evoked neural activities related to perceptual category of syllabic stimuli. The same Broca's area result was observed when the identical MVPA procedure was applied to another dataset that had previously implicated the SMG as an important hub of categorical speech processing (Raizada and Poldrack, 2007). The frontal network has been typically viewed as a region involved in speech production and so our findings naturally invite consideration of auditory-motor integration in speech perception.

\section{Possible roles of Broca's area in speech perception}

Converging evidence from multiple sources has demonstrated that there exists an anterior-posterior distinction within Broca's area, where anterior areas play a role in semantic processes and posterior areas are involved in phonetic and phonological processes (Poldrack et al., 1999; Gold et al., 2005; Gough et al., 2005). Lesion data as well as functional imaging results have suggested a role for pars opercularis (the posterior-most region of Broca's area) in sublexical phonetic processing during speech production (Carreiras et al., 2006; Riecker et al., 2008; Papoutsi et al., 2009). The pars opercularis was the significant region of Broca's area in the present study as well. Thus, it is possible that the same phonetic processes or representations that operate in Broca's area during speech production or decision tasks also participate in sublexical speech perception.

Another possible role for posterior Broca's area in speech perception relates to its role in motor aspects of speech, specifically articulatory planning. Recently, evidence from neuroimaging (Wilson et al., 2004) and transcranial magnetic stimulation (Watkins et al., 2003) studies has suggested that motor systems play a role in speech perception (Pulvermüller et al., 2006; Devlin and Aydelott, 2009). These findings have been also viewed as evidence supporting putative mirror neuron systems in the human brain (D'Ausilio et al., 2009). Relatedly, Skipper et al. (2007) presented evidence that visual contexts (e.g., viewing mouth movement) aid speech perception by recruiting motor systems. Consistent with Skipper et al. (2007), the present study found that a frontal motor-speech network consisting of Broca's area and the left pre-SMA evoked distinct neural patterns corresponding to the perceived syllables. One plausible scenario is that Broca's area generated articulatory representations to guide categorization of the phonetic continua into discrete syllabic units via feedback mechanisms, as proposed in various current speech models (Callan et al., 2004; Davis and Johnsrude, 2007; Rauschecker and Scott, 2009). This articulatory guidance was presumably subconscious in these experiments, as the fMRI task was orthogonal to syllabic identity and subjects were not aware of the behavioral categorization task until afterward.

We also identified pre-SMA activity related to syllable identity. The pre-SMA, which rarely if ever activates in univariate imaging studies of speech perception, has been viewed as a highlevel action-control region mediating sensory-motor transition (Nachev et al., 2008), and has recently received attention in speech production (Alario et al., 2006). Lesions in pre-SMA cause deficits in production of planned speech (Ziegler et al., 1997), suggesting that it plays a role in the preparation of sequential movements, especially in conditions involving a short-term buff- ering of motor-speech acts. Thus, the pre-SMA finding here further supports involvement of articulatory motor processes in speech perception (Pulvermüller et al., 2006). We surmise that articulatory codes and sequences accessed in Broca's area and nearby premotor cortex may be transmitted to the pre-SMA for buffering during speech production or modeling to guide speech perception. In particular, the frontal motor system may play a role when identifying ambiguous or degraded speech signals. For example, a neuroimaging study revealed that, during an / $\mathrm{r} /$ versus / $/$ / consonant identification task, Broca's area was more activated in Japanese speakers whose mother tongue did not differentiate the two consonant sounds than in native English speakers (Callan et al., 2004). Motor systems may also contribute to perception in adverse listening circumstances, as evidenced by the specific perceptual difficulty patients with Broca's aphasia experience when speech signals are degraded (Utman et al., 2001; Moineau et al., 2005). The current findings lend further support to the theory that auditory and motor systems interact during speech perception.

\section{Different spatial scales of processing along the dorsal speech perception stream}

Intriguingly, the MVPA and univariate adaptation analysis of the same dataset (Raizada and Poldrack, 2007) yielded completely different results, the former identifying Broca's area, and the latter identifying SMG. For this new analysis, only the within-category pairs were chosen for binary classification, whereas in the previous study, the neural activities of betweencategory pairs were contrasted with those of within-category pairs. Thus, it is important to underscore that the analyses were not performed for identical comparisons, although both were designed to isolate the same perceptual processes. Recently Zevin and colleagues (2010) demonstrated that the SMG is involved in domain-general change detection, and is thus commonly active during adaptation paradigms regardless of speech content. Hence, the discrepancy between the univariate and multivariate results may reflect different roles for SMG (change detection or discrimination) and Broca's area (phoneme identification) in speech perception.

However, in the visual domain, simultaneous application of a univariate adaptation paradigm (Grill-Spector and Malach, 2001) and a multivariate analysis on the identical data (Drucker and Aguirre, 2009) has also revealed differences in localization of activity, as in our study. This difference may have occurred because local networks in different areas of the brain operate at different spatial scales, reflecting different underlying computational properties. Different types of fMRI analysis reveal different spatial scales of neural processing. Adaptation-fMRI distinguishes between differently tuned populations of neurons that are intermingled within the same voxel, and so highlights processing at a subvoxel spatial scale. In contrast, MVPA reveals areas where information is represented by distributed patterns of activity, thereby highlighting processing at a multivoxel spatial scale. Thus, the findings here may indicate that Broca's area and the SMG use different spatial scales (intervoxel vs intravoxel, respectively) to process speech inputs. Recently, MVPA studies have shown distributed neural patterns for speech perception within large expanses of the temporal lobes (Formisano et al., 2008; Kilian-Hütten et al., 2011). One possible reason for the discrepancy between our main findings and those of previous studies is that auditory representations of phonemes are coded by patterns of activity distributed across large expanses of temporal cortex, while frontal representations are coded in patterns across 
smaller expanses. Thus, the relatively small three-voxel searchlight might be spatially tuned to detect frontal representations, but too small to detect temporal representations. To test this theory, we performed additional searchlight analyses with larger sizes of local spheres. At a radius of eight voxels, the pars opercularis cluster was no longer significant, but the searchlight revealed a significant cluster in the left posterior STG in the new dataset. This finding supports the theory that frontal and temporal speech representations are distributed at different spatial scales. Because of the large size of the searchlights in this analysis $(\sim 21 \mathrm{~mm}$ radius), searchlights contributing to this STG cluster included voxels in the mid-to-posterior STG and middle temporal gyrus, and portions of the SMG. This could be taken as a caveat to our claim that SMG speech processing occurs at a small spatial scale. However, much of the STG cluster was too far from the SMG to have included it in the searchlights. The failure to replicate this finding in the old dataset should also be taken as a caveat, but was likely because unsmoothed images were no longer available and only subsets of the trials in the old dataset were used. However, future studies should also consider alternative explanations for differential spatial scale along the frontotemporal speech network, such as differing hemodynamic response profiles across different regions (Handwerker et al., 2004). Because MVPA are sensitive to coherent patterns of information-carrying activity rather than to univariate intensity differences, it is unlikely that differing hemodynamic response profiles across different regions could account for the current findings. Nonetheless, it would be interesting for future studies to investigate these questions further.

In summary, our results clearly demonstrate the presence of phoneme category-specific information in pars operculars within Broca's area. Further research is needed to clarify the specific role of this area in speech perception. Our findings also raise interesting questions regarding differences in the spatial scale of cortical representations or processes along speech-perception pathways. This principle likely applies to other perceptual domains as well. The possibility that different regions of the cortex encode information at different scales of spatial distribution, or even that a single region may encode different information at different spatial scales, should be considered and examined further in future studies.

\section{References}

Alario FX, Chainay H, Lehericy S, Cohen L (2006) The role of the supplementary motor area (SMA) in word production. Brain Res 1076:129-143.

Callan DE, Jones JA, Callan AM, Akahane-Yamada R (2004) Phonetic perceptual identification by native- and second-language speakers differentially activates brain regions involved with acoustic phonetic processing and those involved with articulatory-auditory/orosensory internal models. Neuroimage 22:1182-1194.

Carreiras M, Mechelli A, Price CJ (2006) Effect of word and syllable frequency on activation during lexical decision and reading aloud. Hum Brain Mapp 27:963-972.

Celsis P, Boulanouar K, Doyon B, Ranjeva JP, Berry I, Nespoulous JL, Chollet F (1999) Differential fMRI responses in the left posterior superior temporal gyrus and left supramarginal gyrus to habituation and change detection in syllables and tones. Neuroimage 9:135-144.

Chang EF, Rieger JW, Johnson K, Berger MS, Barbaro NM, Knight RT (2010) Categorical speech representation in human superior temporal gyrus. Nat Neurosci 13:1428-1432.

D’Ausilio A, Pulvermüller F, Salmas P, Bufalari I, Begliomini C, Fadiga L (2009) The motor somatotopy of speech perception. Curr Biol 19:381-385.

Davis MH, Johnsrude IS (2007) Hearing speech sounds: top-down influences on the interface between audition and speech perception. Hear Res 229:132-147.

Desai R, Liebenthal E, Waldron E, Binder JR (2008) Left posterior temporal regions are sensitive to auditory categorization. J Cogn Neurosci 20:1174-1188.

Devlin JT, Aydelott J (2009) Speech perception: motoric contributions versus the motor theory. Curr Biol 19:R198-R200.

Drucker DM, Aguirre GK (2009) Different spatial scales of shape similarity representation in lateral and ventral LOC. Cereb Cortex 19:2269-2280.

Formisano E, De Martino F, Bonte M, Goebel R (2008) "Who" is saying "what"? Brain-based decoding of human voice and speech. Science 322:970-973.

Gold BT, Balota DA, Kirchhoff BA, Buckner RL (2005) Common and dissociable activation patterns associated with controlled semantic and phonological processing: evidence from FMRI adaptation. Cereb Cortex 15:1438-1450.

Gough PM, Nobre AC, Devlin JT (2005) Dissociating linguistic processes in the left inferior frontal cortex with transcranial magnetic stimulation. J Neurosci 25:8010-8016.

Grill-Spector K, Malach R (2001) fMR-adaptation: a tool for studying the functional properties of human cortical neurons. Acta Psychol (Amst) 107:293-321.

Handwerker DA, Ollinger JM, D'Esposito M (2004) Variation of BOLD hemodynamic responses across subjects and brain regions and their effects on statistical analyses. Neuroimage 21:1639-1651.

Haxby JV, Gobbini MI, Furey ML, Ishai A, Schouten JL, Pietrini P (2001) Distributed and overlapping representations of faces and objects in ventral temporal cortex. Science 293:2425-2430.

Hickok G, Poeppel D (2007) The cortical organization of speech processing. Nat Rev Neurosci 8:393-402.

Husain FT, Fromm SJ, Pursley RH, Hosey LA, Braun AR, Horwitz B (2006) Neural bases of categorization of simple speech and nonspeech sounds. Hum Brain Mapp 27:636-651.

Hutchison ER, Blumstein SE, Myers EB (2008) An event-related fMRI investigation of voice-onset time discrimination. Neuroimage 40:342-352.

Kilian-Hütten N, Valente G, Vroomen J, Formisano E (2011) Auditory cortex encodes the perceptual interpretation of ambiguous sound. J Neurosci 31:1715-1720.

Kriegeskorte N, Goebel R, Bandettini P (2006) Information-based functional brain mapping. Proc Natl Acad Sci U S A 103:3863-3868.

Liberman AM, Mattingly IG (1985) The motor theory of speech perception revised. Cognition 21:1-36.

Liberman AM, Harris KS, Hoffman HS, Griffith BC (1957) The discrimination of speech sounds within and across phoneme boundaries. J Exp Psychol 54:358-368.

Moineau S, Dronkers NF, Bates E (2005) Exploring the processing continuum of single-word comprehension in aphasia. J Speech Lang Hear Res 48:884-896.

Myers EB, Blumstein SE, Walsh E, Eliassen J (2009) Inferior frontal regions underlie the perception of phonetic category invariance. Psychol Sci 20:895-903.

Nachev P, Kennard C, Husain M (2008) Functional role of the supplementary and pre-supplementary motor areas. Nat Rev Neurosci 9:856-869.

Okada K, Rong F, Venezia J, Matchin W, Hsieh IH, Saberi K, Serences JT, Hickok G (2010) Hierarchical organization of human auditory cortex: evidence from acoustic invariance in the response to intelligible speech. Cereb Cortex 20:2486-2495.

Papoutsi M, de Zwart JA, Jansma JM, Pickering MJ, Bednar JA, Horwitz B (2009) From phonemes to articulatory codes: an fMRI study of the role of Broca's area in speech production. Cereb Cortex 19:2156-2165.

Pereira F, Mitchell T, Botvinick M (2009) Machine learning classifiers and fMRI: a tutorial overview. Neuroimage 45:S199-S209.

Poldrack RA, Wagner AD, Prull MW, Desmond JE, Glover GH, Gabrieli JD (1999) Functional specialization for semantic and phonological processing in the left inferior prefrontal cortex. Neuroimage 10:15-35.

Pulvermüller F, Huss M, Kherif F, Moscoso del Prado Martin F, Hauk O, Shtyrov Y (2006) Motor cortex maps articulatory features of speech sounds. Proc Natl Acad Sci U S A 103:7865-7870.

Raizada RD, Poldrack RA (2007) Selective amplification of stimulus differences during categorical processing of speech. Neuron 56:726-740.

Raizada RD, Tsao FM, Liu HM, Kuhl PK (2010) Quantifying the adequacy of neural representations for a cross-language phonetic discrimination task: prediction of individual differences. Cereb Cortex 20:1-12. 
Rauschecker JP, Scott SK (2009) Maps and streams in the auditory cortex: nonhuman primates illuminate human speech processing. Nat Neurosci $12: 718-724$

Riecker A, Brendel B, Ziegler W, Erb M, Ackermann H (2008) The influence of syllable onset complexity and syllable frequency on speech motor control. Brain Lang 107:102-113.

Skipper JI, van Wassenhove V, Nusbaum HC, Small SL (2007) Hearing lips and seeing voices: how cortical areas supporting speech production mediate audiovisual speech perception. Cereb Cortex 17:2387-2399.

Turkeltaub PE, Coslett HB (2010) Localization of sublexical speech perception components. Brain Lang 114:1-15.

Utman JA, Blumstein SE, Sullivan K (2001) Mapping from sound to meaning: reduced lexical activation in Broca's aphasics. Brain Lang 79:444-472.
Watkins KE, Strafella AP, Paus T (2003) Seeing and hearing speech excites the motor system involved in speech production. Neuropsychologia 41:989-994.

Wilson SM, Saygin AP, Sereno MI, Iacoboni M (2004) Listening to speech activates motor areas involved in speech production. Nat Neurosci 7:701-702.

Zevin JD, McCandliss BD (2005) Dishabituation of the BOLD response to speech sounds. Behav Brain Funct 1:4.

Zevin JD, Yang J, Skipper JI, McCandliss BD (2010) Domain general change detection accounts for "dishabituation" effects in temporal-parietal regions in functional magnetic resonance imaging studies of speech perception. J Neurosci 30:1110-1117.

Ziegler W, Kilian B, Deger K (1997) The role of the left mesial frontal cortex in fluent speech: evidence from a case of left supplementary motor area hemorrhage. Neuropsychologia 35:1197-1208. 